\title{
9 Integrierte diagrammatische Methodologie
}

Wo stehen wir nun? In den beiden Teilen Grundlagen und Praktiken versuchte ich, für eine diagrammatische Sichtweise auf linguistische Forschungspraktiken zu plädieren. Es sollte deutlich geworden sein, wie diagrammatische Grundfiguren Untersuchungsgegenstände hervorbringen, wie diagrammatische Praxis von Denkstilen durchdrungen ist und welch entscheidenden Anteil an diesen Operationen der Computer einnimmt, der als diagrammatische Maschine verdatete Sprachgebrauchsdaten transformiert.

Im Fazit geht es mir nun schließlich darum, aus diesen Beobachtungen und Analysen eine diagrammatische Methodologie zu synthetisieren. Diese Methodologie soll aber keine Handlungsanweisung sein, um „bessere“ Diagramme zu erzeugen oder eine Evaluationsform vorzuschlagen, um Diagramme zu evaluieren. Sie soll eher als Orientierungsrahmen verstanden werden, mit dem die linguistische diagrammatische Praxis besser reflektiert werden kann.

Ich werde zunächst nochmals die Bandbreite diagrammatischen Operierens in sprachwissenschaftlichen Kontexten aufgrund der bisher gemachten Beobachtungen und Überlegungen aufspannen. Danach geht es um die Frage, wie dieser methodologische Orientierungsrahmen diagrammatischer Praxis so gewendet werden kann, dass damit ein spezifisches Problem eines Fachs in den Geisteswissenschaften, das Methoden anderer Disziplinen verwendet, beleuchtet: Nämlich das Verhältnis von maschinellen Analysen und Interpretation.

Und schließlich möchte ich eine provokante Richtung einschlagen und dafür optieren, neue diagrammatische Transformationen zu suchen, die gegen Denkstile und entsprechende Kanons verstoßen und quer zur Praxis liegen, um neue Untersuchungsgegenstände $\mathrm{zu}$ formen und damit neue Sinnangebote zu schaffen.

\subsection{Diagrammatische Operationen zwischen Code und Interpretation}

Diagrammatische Transformationen, in einem umfassenden Sinn verstanden, der auch Datentransformationen umfasst, sind Dreh- und Angelpunkte wissenschaftlichen Arbeitens. Ein linguistisches Forschungsvorhaben geht meist auch mit einer impliziten oder expliziten Entscheidung für eine diagrammatische Grundfigur (Kapitel 5) einher, mit der Daten im Jäger’schen Sinn ein erstes Mal transkribiert und damit deutbar gemacht werden. Dabei kommt es zu Effekten, die für sprachliche Daten mit Rekontextualisierungen, Desequenzialisierungen, Dimensionsanreicherungen und Rematerialisierungen gefasst werden

๖ Open Access. (C) 2020 Noah Bubenhofer, publiziert von De Gruyter. (c) BY Dieses Werk ist lizenziert unter der Commons Attribution 4.0 Lizenz.

https://doi.org/10.1515/9783110698732-009 
können (Abschnitt 5.6). Diese Effekte können gezielt genutzt werden, um durch die Transkription bestimmte Aspekte der Daten hervortreten oder verschwinden zu lassen. Werden diese Effekte jedoch nicht erkannt und reflektiert, führen die diagrammatischen Transformationen zu Simplifizierungen der Daten. Für die Linguistik ist beispielsweise der Effekt der Desequenzialisierung von entscheidender Bedeutung: Viele diagrammatischen Transformationen brechen die Sequenzialität von sprachlichen Äußerungen auf und behandeln sie als „Bag of Words“. Viele Methoden nutzen genau diese Konzeption von Text oder Gespräch zusammengefasst als Sack von Wörtern (oder andere sprachlichen Einheiten) und ermöglichen dafür eine Reihe von Transformationen, die ansonsten nicht möglich wären.

Der Verlust einer Eigenschaft wie Sequenzialität geht jedoch mit einem Verlust an Perspektiven einher. Mit einem „Bag of Words“-Ansatz sind Fragen nach Dynamik innerhalb des Sackes, also des Textes, müßig - solche Methoden präferieren eine Konzeption von Sprache, bei der Texte, oder die Einheit, die als Sack aufgefasst wird, als kleinste zu interessierende Einheit definiert wird. Die Binnenstruktur innerhalb eines Textes ist dabei nicht mehr von Belang.

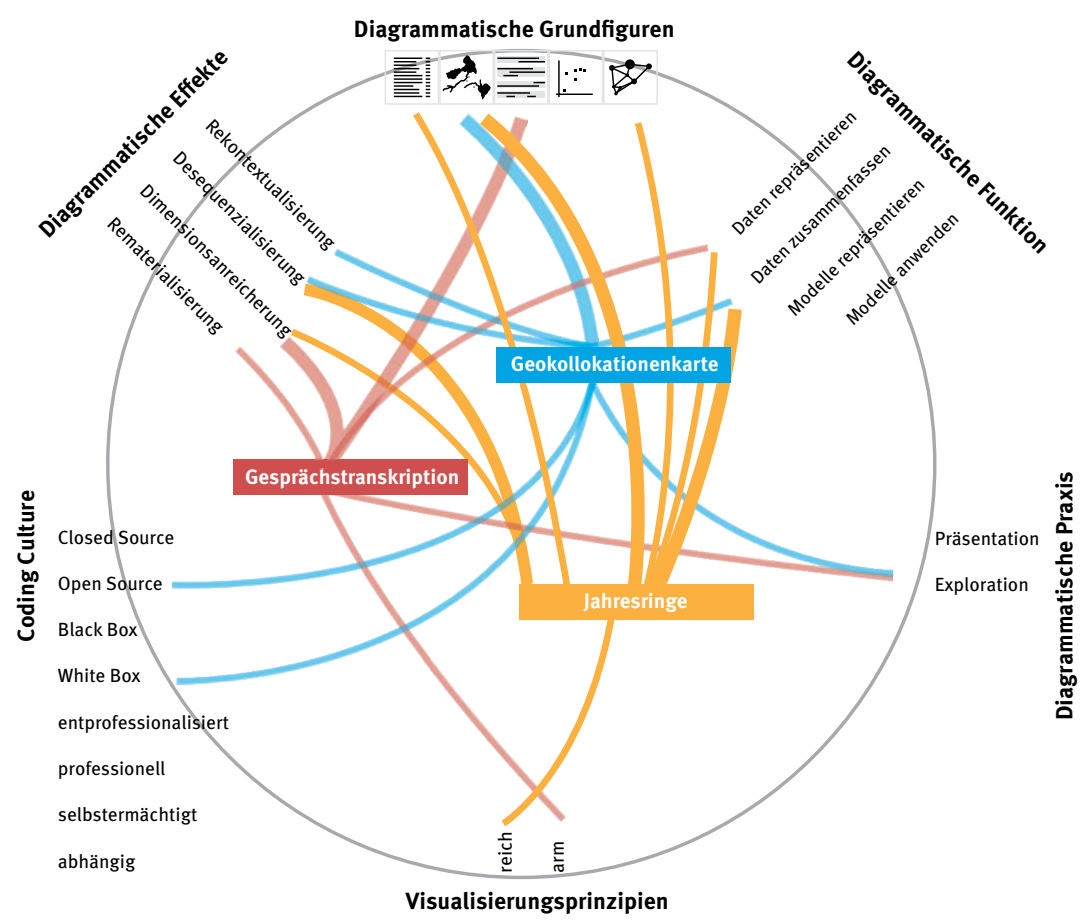

Abb. 110: Integrales Modell diagrammatischen Operierens in der Linguistik 
Visualisierungen ermöglichen unterschiedliche diagrammatische Praktiken: Entweder dienen sie eher der Präsentation oder aber der Exploration von Daten. Diagrammatische Operationen entfalten sich besonders dann, wenn die Visualisierung darauf angelegt ist, Daten überblickbar und damit in Bezug auf bestimmte Aspekte überhaupt erst analysierbar zu machen, sie also als Werkzeug dienen, um Transformationen und Interpretationen vorzunehmen.

Die Diagramme erfüllen darüber hinaus unterschiedliche Repräsentationsfunktionen: Sie können Daten zeigen oder zusammenfassen oder aber Modelle repräsentieren oder Modelle anwenden.

Bei Visualisierungen, die mit Hilfe des Computers entstehen oder gar in die diagrammatischen Prozesse der Datentransformationen komplett eingebunden sind, spielen Coding Cultures eine wichtige Rolle bei den Transformationsprozessen. Man kann sich in einer Hacking-Kultur bewegen, die von Open Source, White Box, Entprofessionalisierung und Selbstermächtigung geprägt ist - oder in einer kommerziell-proprietären Kultur, sowie allen möglichen Zwischenformen. Je nach Coding Culture, in der man sich bewegt, entstehen unterschiedliche Visualisierungen, die unterschiedliche diagrammatische Transformationen nahelegen. Bewegt man sich beispielsweise in der Open Source Javascript-Kultur und sieht in den zu analysierenden Daten eine Netzstruktur, ist es wahrscheinlich, dass eine der verfügbaren Javascript-Bibliotheken genutzt wird, die bestimmte Programmiertechniken, Funktionen und auch eine bestimmte Ästhetik nahelegen. Es ist also nur in wenigen Fällen so, dass die Analyse des Visualisierungsproblems und die sich daraus ergebenden Anforderungen an die technische und gestalterische Umsetzung als zwei getrennte und aufeinander folgende Schritte praktiziert werden. Die Coding Culture, in der man sich bewegt, nimmt das Ergebnis der Visualisierung bis zu einem gewissen Grad vorweg.

Das Zusammenspiel von Daten, diagrammatischer Grundfigur und Coding Culture führt dann zu Visualisierungsprinzipien, die eher reich oder arm sind, also einen mehr oder weniger großen Möglichkeitsraum der Operationen und Transformationen zulassen. Reiche Visualisierungsprinzipien haben dabei eher das Potenzial, Kanons und Denkstile zu durchbrechen. Ärmere Visualisierungsprinzipien hingegen bewegen sich eher im Kanon und damit im Denkstil der Disziplin.

Einzelne Diagramme können nun innerhalb dieses Modells verschiedener Aspekte verortet werden. Abbildung 110 zeigt ein Diagramm, mit dem Diagramme bezüglich der herausgearbeiteten Kriterien verortet werden können. Es geht dabei aber nicht darum, allgemeingültige Klassifikationen von Diagrammen festzulegen, sondern das Modell und dessen diagrammatische Gestalt dienen als Wegweiser für eigene Fragestellungen, in die diagrammatische Aspekte involviert sind. Hinsichtlich einer reflexiven Haltung gegenüber dem eigenen diagramma- 
tischen Vorgehen zeigt es die Aspekte, die bedacht werden müssen. Damit ist immer auch die Arbitrarität der jeweiligen Position im Modell verbunden, denn: Es gibt immer eine Wahl, die Visualisierung bezüglich der genannten Aspekte auch anders zu erstellen. Das Modell zeigt, wie man zu den möglichen Alternativen gelangen kann.

Ein Beispiel soll das illustrieren: Für eine gesprächslinguistische Fragestellung entscheidet man sich dazu, die Daten zu transkribieren. Das geschieht in Partiturschreibweise mittels des Editors EXMARaLDA und nach der Transkriptionsrichtlinie GAT 2. Im Modell verortet sich ein solches Transkript bei folgenden Ausprägungen der Kategorien:

- Diagrammatische Grundfigur: Partitur

- Diagrammatische Effekte:

- Dimensionsanreicherung

- Rematerialisierung

- Diagrammatische Funktion: Daten repräsentieren

- Diagrammatische Praxis: Exploration

- Visualisierungsprinzip: eher arm

- Coding Culture:

- Closed Source (der Quellcode des Programms ist nicht öffentlich)

- eher White Box (die Funktionsweise ist - obwohl der Quellcode nicht öffentlich ist - relativ transparent)

- professionell (Einschätzung von außen, da der Quellcode nicht öffentlich ist)

- abhängig (bei der Benutzung ist man an das grafische Benutzerinterface gebunden)

Selbstverständlich ist diese Verortung diskussionswürdig. Insbesondere die Frage, ob der Möglichkeitsraum für weitere Transformationen und Operationen des Visualisierungsprinzips eher arm oder reich ist, kann keine klar zu entscheidende Frage sein. Dass ich das Gesprächstranskript als „eher armes“ Visualisierungsprinzip charakterisieren würde, liegt darin begründet, dass in der Praxis die diagrammatische Grundfigur der Partitur in keinem Fall aufgebrochen oder ergänzt wird, gerade auch, wenn Audio- oder Videospuren damit aligniert werden. Wichtig ist jedoch weniger Einigkeit über die Einschätzung. Wichtiger ist, dass die Kategorien einen dazu zwingen, das Diagramm unter diesen Aspekten $\mathrm{zu}$ bewerten und damit die ganze Bandbreite der Faktoren, die die diagrammatische Praxis beeinflussen, deutlich wird.

In Abbildung 110 sind neben dem klassischen Gesprächstranskript im Kontrast dazu das Jahresringe-Diagramm (vgl. Abschnitt 8.2.2) und die Geokollokationen-Anwendung (vgl. Abschnitt 6.2.3) eingetragen. Aus Gründen der 
Übersichtlichkeit werden nicht alle zutreffenden Aspekte den Diagrammtypen zugewiesen, sondern nur für den jeweiligen Typus besonders relevante. Und zudem habe ich einige besonders wichtige Aspekte durch eine stärkere Strichdicke hervorgehoben. Nun ist beispielsweise ersichtlich, dass das JahresringeDiagramm auf mehrere diagrammatische Grundfiguren zurückzuführen ist, was beim Gesprächstranskript nicht der Fall ist.

Wenn ein Diagramm bezüglich der herausgearbeiteten Aspekte verortet wird, können danach alternative Diagramme abgeleitet werden, die bezüglich bestimmter Aspekte, also bezüglich Effekte, Funktionen, diagrammatischer Praxis etc., anders zu charaktersieren sind. Dies ist mit der These verbunden, dass eine Alternative zu einem Diagramm erst dann entsteht, wenn mindestens einer dieser Aspekte anders gesetzt wird. Im Fall des Gesprächstranskripts wäre etwa zu überlegen, ob eine Desequenzialisierung (als Ergänzung) nicht auch ein wünschenswerter Effekt wäre, der einen neuen Blick auf die Daten ermöglichen würde. Oder es wäre zu prüfen, ob die Funktion der Datenzusammenfassung nicht interessante Erkenntnisse bringen würde. Damit werden jeweils andere diagrammatische Grundfiguren und damit Visualisierungsprinzipien möglich und es kommt zu einer anderen Art der Gegenstandskonstitution. DiagrammAlternativen, die die gleichen Aspekte nutzen, führen nicht zu neuen Visualisierungsprinzipien und von ihnen ist auch kein bedeutender Einfluss auf die Gegenstandskonstitution zu erwarten.

Es versteht sich von selbst, dass solche Entscheidungen Hand in Hand mit den Forschungsfragen, die verfolgt werden sollen, gehen. Hand in Hand bedeutet in diesem Fall aber auch, dass nicht zwingend die Forschungsfrage voraus gehen muss, und davon das Diagramm abgeleitet wird, sondern dass auch der umgekehrte Weg gegangen werden kann, gerade, wenn es konkret darum geht, herrschende Denkstile in Frage zu stellen oder zu umgehen.

\subsection{Daten deuten und verstehen}

Die Transformationen von mittels Computer Verdatetem ähneln sich, egal ob es sich beim Verdateten um Sprachdaten, Messwerte oder Umfragedaten handelt. Das ist der Clou des Metamediums Computer. Auch die Formen der Formatierung und Visualisierung auf Bildschirmen, Druckern, 3D-Brillen etc. ähneln sich oft. Es ist deswegen naheliegend, dass sich der Sprachwissenschaftler mit der Informatikerin, dem Computerlinguisten, der Textminerin und der Statistikerin austauscht, um gegenseitig Methoden, Werkzeuge und Programmcode zu nutzen. Wir selbst gingen so vor, um Korpora aufzubereiten, auszuzeichnen und auszuwerten. 
Mit Fleck gesprochen unterscheiden sich Disziplinen oder Bereiche aber teilweise markant durch ihre unterschiedlichen Denkstile und Forschungslogiken. Ein Beispiel seien zwei eigentlich nahe Disziplinen: Linguistik (bis hin zu Korpuslinguistik) und Computerlinguistik. Deutlich werden die unterschiedlichen Denkstile an einer Fragestellung, die beide Disziplinen beschäftigt: Autorschaftsattribution. Die Frage ist also, welche Merkmale eines Textes typisch für den Stil eines Autors oder einer Autorin sind um einen Text mit unbekannter Autorschaft einer Person zuordnen zu können. Typischerweise ist zur Beantwortung dieser Frage das folgende Setting üblich: Es gibt eine Menge von Texten mit bekannter Autorschaft. Aufgrund dieser Texte trainiert man ein statistisches Modell, das die Autorschaft für einen Text voraussagen kann. Dieses Modell evaluiert man an Texten, deren Autorschaft bekannt ist und kann es dann an Texten mit unbekannter Autorschaft anwenden, um eine Prognose über die Autorschaft abzugeben.

Die Kalibration dieses Settings erfolgt über die Merkmale („Features“), mit denen das Modell trainiert werden soll, sowie über die Auswahl des statistischen Modells. Diagrammatisch gesehen werden die Texte in einen Vektorraum überführt, indem die Häufigkeiten der Merkmalsausprägungen pro Text gemessen werden. Ein einfaches Beispiel sind Wortformen: Ein Text wird in einen Vektor überführt, der die (ggf. gewichteten) Häufigkeiten aller Wortformen im Korpus repräsentiert. Aus diagrammatischer Perspektive wird zunächst ein Index der vorhandenen Wortformen (diagrammatische Grundform der Liste, Effekt der Rekontextualisierung) und daraus die Vektorrepräsentation erstellt (diagrammatische Grundform Vektor). Dies ist gleichzeitig ein weiterer Schritt der Verdatung, da nun Texte als Zahlenvektoren repräsentiert werden können (vgl. dazu für Details Abschnitt 4.1). Nun können im Vektorraum Ähnlichkeiten der Texte berechnet werden, indem die Distanz zwischen den Vektoren berechnet wird. Und natürlich kann der Vektorraum in ein grafisches Diagramm formatiert werden, wobei dabei eine Dimensionsreduktion vom n-dimensionalen Raum ( $\mathrm{n}=$ Anzahl Wortformen-Types) in zwei oder allenfalls drei Dimensionen gemacht werden muss, um die Vektoren in einem x-y- (ggf. z-)Koordinatenraum auf einer Fläche darstellen zu können. Im Vektorraum müssten sich im Idealfall dann Texte der gleichen Autorschaft clustern, d.h., die Vektoren dieser Texte müssten in eine ähnliche Richtung zeigen. Die Feststellung dieser Cluster kann dann wiederum ein Algorithmus übernehmen, der dann eben typische Vektorrepräsentationen für alle Texte eines Autors/einer Autorin modelliert. Der Vektor eines Textes mit unbekannter Autorschaft kann nun in der Distanz zu den typischen Vektoren verortet werden - wenn er genug nahe an einem typischen Vektor der Texte eines bekannten Autors/einer bekannten Autorin ist, kann nun - je nach Distanz - sicherer oder weniger sicher die Autorschaft prognostiziert werden. 
Wortformen generell und alleine sind aus linguistischer Sicht keine ausreichenden Merkmale, um den Autorschaftsstil zu bestimmen. Denkbar wäre, zusätzlich Satzkomplexität, Wortwahl in einem semantischen Paradigma, orthographische Varianten oder morphosyntaktische Auffälligkeiten zu modellieren, also Merkmale, die komplexer sind als Wortformen und theoretische Annahmen der Linguistik berücksichtigen.

Besonders erfolgreich ist bei vielen Aufgaben der Autorschaftsattribution aber ein sehr viel einfacheres Merkmal: „low-level features like character n-grams are very successful for representing texts for stylistic purposes“(Stamatatos 2009, 24). Buchstaben-n-Gramme, Sequenzen von Buchstaben, genügen offensichtlich zur Modellierung von Stil, wobei es sich also um ein Merkmal handelt, für dessen Bestimmung kein linguistisches Wissen relevant ist.

Der Erfolg dieses Merkmals kann mittels Evaluation an einem Goldstandard, also einem manuell klassifizierten Datensatz, nachgewiesen werden. Dabei werden normalerweise Präzisions- und Ausbeute-Werte berechnet. Die Werte sagen aus, wie präzise das Modell ist (also wie viele der einem Autor / einer Autorin zugewiesenen Texte richtig zugewiesen worden sind) und wie gut die Ausbeute, also wie viele der zu einem Autor / einer Autorin gehörenden Texte gefunden worden sind. Wenn sowohl Präzision als auch Ausbeute hoch sind, wird davon ausgegangen, dass das Modell präzise ist und nicht-klassifizierte Texte sicher klassifzieren kann.

Funktioniert das Modell, können darauf aufbauend Werkzeuge konstruiert werden, etwa eines, das die Autorschaft von Erpresserbriefen prognostiziert und so Gerichtsfälle lösen kann. Die Konstruktion solcher Werkzeuge - natürlich auch für ganz andere Zwecke - ist normalerweise das Interesse in der Computerlinguistik. Aus linguistischer Perspektive hingegen mag aber primär Verwunderung darüber empfunden werden, dass ein statistisches Modell auf der Basis von Buchstaben-n-Grammen überhaupt funktioniert. Daraus ergibt sich die Frage, was mit Buchstaben-n-Grammen eigentlich gemessen wird. Das ist aber unklar, denn Buchstaben-n-Gramme könnten bestimmte Wortpräferenzen abbilden, die zufälligerweise über ein Buchstaben-n-Gramm erfasst werden. Es könnten auch bestimmte Flexionsendungen sein, die wiederum typisch für bestimmte morphosyntaktische Merkmale sind oder es könnte sogar Satzkomplexität mitgemessen werden, da ein bestimmtes n-Gramm eine Konjunktion repräsentiert. Während diese Aspekte als Ausdruck von Autorschaftsstil angesehen werden können, drücken die Buchstaben-n-Gramme aber auch Themen oder Textsorten aus, die nicht als stilistische Ausprägungen aufgefasst werden können. Die Buchstaben$\mathrm{n}$-Gramme repräsentieren also einen bunten Strauss unterschiedlicher Textmerkmale, von denen nicht alle als Ausdruck von Autorschaftsstil angesehen werden können, wobei aber nicht mal bekannt ist, welche der genannten Aspekte über- 
haupt eine Rolle spielen. Das Modell ist also nicht valide, da unklar ist, was es misst.

Wenn ein Modell primär als Bestandteil eines Werkzeugs benutzt wird, das eine klar umrissene Aufgabe hat, mag fehlende Validität verschmerzbar sein. Ein solcher Fall ist die automatische Erkennung von Spam-E-Mail-Nachrichten, bei der eine statistische Modellierung von Spam-Nachrichten verwendet wird. Aber bei einem linguistischen Interesse auf ein solches Phänomen ist Validität wichtig, um das Ergebnis interpretieren zu können. ${ }^{101}$ Ein statistisches Modell dient im Rahmen einer linguistischen Forschungsfrage dazu, eine Zusammenfassung der Daten auf einer generalisierbaren Ebene zu leisten, aber so, dass das Modell deutbar ist. Dafür muss dessen Funktionsweise und die entscheidenden Merkmalsausprägungen zur Beschreibung der Daten bekannt sein. Damit werden die unterschiedlichen Interessen in Bezug auf die Analyse sprachlicher Daten deutlich sichtbar: Einerseits geht es darum, die Daten deuten und verstehen zu können, andererseits darum, beschreiben und prognostizieren zu können.

Aus geisteswissenschaftlicher Position wird damit deutlich, dass ein Ergebnis maschineller Datentransformation und statistischer Modellierung - mit Jäger gesprochen - ein neues Script darstellt, das genauso gedeutet werden muss, wie ein Text, der vielleicht am Anfang der ganzen Transformationskette stand. Um dies erfolgreich machen zu können, müssen die entstandenen Daten als Ergebnis von diagrammatischen Transformationen verstanden werden können. Da diagrammatische Transformationen und die damit möglichen Operationen gegenstandskonstituierend sind, muss reflektiert werden können, welche Gegenstände mit den Analysen entstanden sind und weiter interpretiert werden. Aus geisteswissenschaftlicher Sicht ist es also elementar, Methoden, die beispielsweise in der Statistik, Informatik oder Computerlinguistik unter anderen wissenschaftstheoretischen Prämissen entwickelt worden sind, diagrammatisch zu verstehen und abzuschätzen, ob sie für das jeweilige Untersuchungsinteresse geeignet sind.

101 Auch in vielen weiteren Bereichen ist Validität statistischer Methoden wichtig. Man denke im Fall der Autorschaftsattribution an die Verwendung dieser Methode vor Gericht, um beispielsweise ein Erpresserschreiben einer verdächtigen Person zuschreiben zu können. Es wäre moralisch nicht vertretbar, eine Person auf der Basis eines statistischen Modells zu verurteilen, das nicht valide ist, also nicht verstanden wird. Vgl. für eine kritische Diskussion verschiedener Methoden zur Autorschaftsattribution aus korpuslinguistischer Sicht die Blogbeiträge von Joachim Scharloth: http://www.security-informatics.de/blog/?tag=authorship-detection (22. 9. 2020) und generell zur Gefahr von Black-Box-Systemen des maschinellen Lernens aus gesellschaftlicher Perspektive O’Neil (2016). Ferner gehen Bubenhofer (2018e) und Bubenhofer / Dreesen (2018) auf den weiteren Zusammenhang von Black-Box-Systemen aus linguistischer Perspektive ein. 


\subsection{Chancen für neue Transformationen}

Zuletzt möchte ich die Frage nach dem innovativen Potenzial diagrammatischer Operationen stellen. Hierzu sei Krämer zitiert:

Inskribierte Flächen machen nicht nur sichtbar, sie machen höchst erfinderisch. Die Einbildungskraft findet in diesem artifiziellen Sonderraum einen intersubjektiv teilbaren Ort. (Krämer 2016, 17)

Je stärker der Möglichkeitsraum eines Visualisierungsprinzips beschränkt wird durch einen, einem bestimmten Denkstil verhafteten, Kanon, desto weniger kann von der Erfindungskraft der inskribierten Fläche profitiert werden. Diesen Zusammenhang sieht auch Mark Lauersdorf und verweist dabei sogar auf eine Gefahr, die von ikonisch gewordenen Visualisierungen ausgeht:

[...] we would be wise to question ourselves regarding some of the standard visualizations that have become iconic in our areas of study. Have we objectified these visualizations thereby fixing (locking in) their form and rendering them immutable/unchangeable (iconic)? And do we interpret all new information and arguments through these fixed forms, potentially focusing, in our subsequent analyses, more on the visualization than on the information behind it? And what are we missing if we are, in fact, doing this? (Lauersdorf 2018, 109)

Lauersdorf macht also auf die Gefahr aufmerksam, dass ikonisch gewordene Visualisierungen zu starren Analyseinstrumenten werden, die bestimmte Interpretationen bevorzugt entstehen lassen. Solche Visualisierungen machen es schwer, die dahinterliegenden Daten oder Informationen anders zu interpretieren, als es die Visualisierung nahelegt. Dies gilt für ein einzelnes Diagramm genau so wie für ein Visualisierungsprinzip, das für verschiedene Darstellungen immer wieder herangezogen wird. Es befördert einen bestimmten Denkstil und damit wird eine andere Sicht des Problems erschwert.

Lauersdorf stellt deswegen eine einfache Forderung: „Use all the data!“ (Lauersdorf 2018) Denn, so sein Argument, werden für eine linguistische Fragestellung alle verfügbaren Daten verwendet, ergeben sich daraus verschiedene Probleme des Datenhandlings, die gelöst werden müssen. Es müssen statistische Verfahren angewandt werden, um spezifische Aspekte zu testen, da die Daten nicht mehr manuell überblickt werden können. Und die Daten müssen visualisiert werden. Es handelt sich also zum großen Teil um diagrammatische Operationen und Transformationen, die nötig sind, um die facettenreiche Datengrundlage prozessieren zu können. Und dabei wird eine Kette von Handlungsaufforderungen ausgelöst, die Lauersdorf wie folgt beschreibt: 
$\rightarrow$ Use all the data.

$\rightarrow$ If you use all the data, view all the data.

$\rightarrow$ If you view all the data, view all the combinations.

$\rightarrow$ If you view all the data, view all the angles.

$\rightarrow$ If you view all the data, use all the techniques. (Lauersdorf 2018, 112)

Die Forderung zielt also darauf ab, das Visualisierungsprinzip möglichst auszureizen - aber eigentlich auch mehr zu tun, nämlich alle Aspekte, die ich im Verlauf der vorliegenden Arbeit als relevant für die diagrammatische Praxis erkannt habe, zu reflektieren und damit zu operieren. Das bedeutet, um die Aspekte nochmals in aller Kürze zusammenzufassen:

- Diagramme lassen sich auf bestimmte diagrammatische Grundfiguren zurückführen. Für die Linguistik identifizierte ich die folgenden Grundfiguren als besonders relevant: Listen, Karten, Partituren, Vektoren und Graphen. Sie transformieren je auf ihre eigene Weise Daten und wirken gegenstandskonstituierend (Kapitel 5).

- Diagramme sind, wie alle Medien, transkriptive Verfahren im Sinne Ludwig Jägers, d.h., sie sind ein sinninszenierendes Verfahren der kulturellen Semantik, mit denen mediale Übersetzungen, Umgestaltungen und Umformungen, also Transformationen, möglich werden. Durch diese Remediatisierung werden Diagramme und Daten neu semantisiert (Abschnitt 3.1).

- Diagrammatische Transformationen führen zu bestimmten Effekten, die sich auf die Daten auswirken. Es handelt sich dabei um Effekte der Rekontextualisierung, Desequenzialisierung, Dimensionsanreicherung und Rematerialisierung. Je nach Untersuchungsinteresse sind diese Effekte erwünscht oder hinderlich (Abschnitt 5.6).

- In der diagrammatischen Praxis werden Diagramme entweder zur Präsentation von Analyseergebnissen oder aber eher zur Exploration von Daten, mit denen diese überhaupt erst analysierbar gemacht werden, verwendet. Dabei ist aber zu bedenken, dass mit der Wahl bestimmter diagrammatischer Mittel der zu untersuchende Gegenstand auch immer auf eine bestimmte Weise mitkonstituiert wird (Abschnitt 2.3.1).

- Digitale, mit Programmcode erstellte Diagramme, werden im Rahmen einer bestimmten Coding Culture erstellt und verwendet. Die Wahl einer bestimmten Programmierumgebung, einer bestimmten Programmiersprache oder Bibliothek erfolgt nicht primär auf der Basis technischer Erwägungen, sondern ist durch die Coding Culture, in der man sich bewegt, geprägt. Die Coding Culture legt Aspekte fest wie Closed vs. Open Source, Black-Box- vs. White-Box-Verfahren, professionelle Implementierung oder Entprofessionalisierung sowie Abhängigkeit von Computern, Institutionen oder Konzernen vs. Selbstermächtigung (Abschnitt 4.4). 
- Das Operieren mit Diagrammen in Form von Grafiken oder aber in Form von digitalen Repräsentationen, geht bei mit Programmcode erstellten Diagrammen ineinander über. Diagramme sind deshalb nicht das Endergebnis einer Datenanalyse, sondern diagrammatisches Operieren ist fester Teil jeglicher Datenanalyse von Beginn weg und bestimmt den Möglichkeitsraum der Transformationen mit (Abschnitt 4.3).

- Die Kombination einer bestimmten diagrammatischen Grundfigur mit einem bestimmten Datentyp und bestimmten Transformationsverfahren ergibt ein Visualisierungsprinzip. Visualisierungsprinzipien können eher enge oder aber weite Möglichkeitsräume für diagrammatische Transformationen eröffnen, so dass von armen und reichen Visualisierungsprinzipien gesprochen werden kann (Abschnitt 5.7).

- Diagrammatische Praktiken sind in allgemeinere wissenschaftliche Praktiken eingebettet, die wiederum Ausdruck von wissenschaftlichen Denkstilen sind. Diese Denkstile führen $\mathrm{zu}$ Kanonisierungen der diagrammatischen Praxis, die in Frage gestellt werden muss, wenn neue, innovative Visualisierungsformen und damit auch Gegenstandskonstitutionen und Perspektiven auf Sprache gewonnen werden sollen (Abschnitt 3.2 und 3.3).

Diese Aspekte definieren Angriffspunkte, um neue diagrammatische Praktiken $\mathrm{zu}$ finden. Also wären - wie bereits beschrieben - Visualisierungen auf der Basis anderer diagrammatischer Grundfiguren zu wählen, andere Effekte zu nutzen, um andere Aspekte der Daten zu repräsentieren und eine andere Programmiersprache oder Programmierumgebung auszuprobieren, um die Algorithmen für die Visualisierung zu implementieren.

Das ist im Forschungsalltag in dieser Konsequenz nicht möglich. Ein Schritt, diesem Ideal näher zu kommen, ist das Hacking-Paradigma in die Wissenschaftspraxis zu übernehmen. Dies bedeutet: Do it yourself, teilen, voneinander lernen, experimentieren und zweckentfremden.

\subsection{Ausblick}

Welche grundlegenderen Folgen ergeben sich nun aus diesen Erkenntnissen für die Linguistik selber, jedoch auch für die benachbarten Disziplinen und die Diagrammatologie? Zum Abschluss mögen die folgenden Beispiele und Überlegungen zu weiteren Ideen führen. 


\subsubsection{Neue diagrammatiko-linguistische, transsemiotische Perspektiven auf Sprachgebrauch}

Der Iconic Turn auf der Basis von Überlegungen von William J. T. Mitchell (1992, wiederabgedruckt 2013) und Gottfried Böhm (1994) erhofft sich einen zweifachen Nutzen des Fokus auf Bildlichkeit: Einerseits soll das Bildhafte an kulturellem Handeln anerkannt werden, andererseits sollen aber Bilder auch als Analyseinstrument von Kultur genutzt werden können: Bilder sind nicht nur „Zeichen, Abbilder oder Illustrationen; sie entfalten eine ganz eigene Wirkungsmacht, die sich der Sprache zu entziehen scheint“ (Bachmann-Medick 2006, 331). Ein Element dieser Wirkungsmacht (die ich im Buch mehrfach diskutiert habe) ist das erkenntnisfördernde Potenzial von Bildern:

Wenn es im iconic turn ausdrücklich nicht nur darum gehen soll, Bilder zu verstehen, sondern die Welt durch Bilder zu verstehen, lässt sich also hier erst unter einer Grundbedingung von einem turn sprechen: dass die Gegenstandsebene (d.h. Bilder als Untersuchungsgegenstand) gewissermaßen umschwenkt auf die Ebene der methodischen Einstellungen, dass Bilder somit selbst als Erkenntnismedien und Analysekategorien in den Blick kommen. (Bachmann-Medick 2006, 350)

Wenn nun die Linguistik die Ideen des Iconic Turn produktiv aufnehmen möchte, ist der erste Schritt sicher die Integration von Medialität und Multimodalität in die linguistischen Analysen. Um aber das Postulat des Iconic Turn ernst zu nehmen und Bilder als Erkenntnismedien zu nutzen, ist eine diagrammatische Perspektive auf Sprachgebrauch ein wichtiger Schlüssel - und zwar gerade nicht nur im engen Sinn als Bildlichkeit verstanden, sondern als semiotisch komplexe Konfigurationen von Schriftlichkeit und Bildlichkeit, als transkriptive, sinninszenierende Verfahren. Ein Beispiel mag das Potenzial zeigen:

Interessiert man sich etwa für Social Media und hegt ein diskursanalytisches Interesse an (sprachlichen / kommunikativen) Praktiken darin, zeigt sich die diagrammatische Perspektive als hilfreich (Bubenhofer 2019). So zeigen sich die Inhalte in den Interfaces von Twitter, Facebook, Instagram etc. als (teilweise schwach hierarchisierte) Listen, wobei mit dem Taggen eine Art Glossierung und Kategorisierung der Beiträge erreicht wird. Die Praktik des Taggens ist linguistisch beispielsweise interessant, da es gattungskonstitutiv wirken kann, wie Meier-Vieracker anhand der „Wutrede“ - öffentliche, aber spontane, emotionalimpulsive Redebeiträge von Funktionsträgern wie Fußballtrainern - zeigte (Meier 2016, 20). Aus diagrammatischer Perspektive zeigen sich aber verschiedene Diskrepanzen zwischen der Erscheinungsform der Inhalte auf Sozialen Medien als Listen und den damit verbundenen menschlichen und technischen Operationen (Bubenhofer 2019, 125): 
- Es sind nicht nur menschliche Annotationen, die zu Kategorisierungen, wie eben im Beispiel von Videobeiträgen als „Wutreden“, führen, sondern es handelt sich um eine Vermischung von menschlichen und algorithmischen Ordnungspraktiken.

- Während die Inhalte den Nutzenden als „Streams“, also algorithmisch geordneten Listen, erscheinen, verbirgt sich dahinter eine netzwerkartige Struktur als Ergebnis von Interaktionen: Nutzende können einander folgen, Texte sind intertextuell über die Tags verknüpft und Algorithmen gruppieren Texte und Nutzende nach maschinell erkannter Ähnlichkeit. Dies ermöglicht es den Plattformbetreibern Individuen $\mathrm{zu}$ profilieren und Voraussagen über zukünftiges Verhalten zu treffen. Die Listen und Streams verdecken die kommerziell interessante diagrammatische Grundfigur des Netzwerkgraphen im Hintergrund.

Diese Beobachtungen sind nicht nur diskursanalytisch interessant, sondern stellen auch methodologische Probleme. Sollen z. B. Daten aus Social Media korpuslinguistisch untersucht werden, müssen Datensätze als Samples aus den Datenströmen gewonnen werden. Doch welche Datenströme werden berücksichtigt? Was sollte überhaupt als Text aufgefasst werden, wenn man davon ausgeht, dass jede Nutzerin und jeder Nutzer eine individuelle Liste von Nachrichten präsentiert erhält? Jede spezifische Liste als Konfiguration von annotierten und verschieden kategorisierbaren Minitexten stellt eine immer wieder neue Rekontextualisierung dar. Die potenziellen „Listentexte“ müssten analytisch rekonstruiert werden, um bei der Analyse nicht bei einer Menge von dekontextualisierten Einzelnachrichten stecken zu bleiben, sondern die rekontextualisierenden Kräfte der verschiedenen Listen sichtbar zu machen. Zudem muss analytisch entschieden werden, ob die Daten eher als Listen betrachtet oder ob die netzwerkartige Struktur rekonstruiert werden soll. Somit ist klar, dass die Analyse selber eine diagrammatische Operation ist - eine Operation freilich, die nicht nur wir als Forschende, sondern der Plattformbetreiber selber ebenfalls macht, um die Plattform kommerziell zu optimieren (Bubenhofer 2019, 131-132).

\subsubsection{Coding Cultures, Technikkulturen, Praktiken, Gender}

In Kapitel 4.4 habe ich auf die Coding Cultures, also Praktiken des Programmierens aufmerksam gemacht, die für algorithmisch erstellte Diagramme von großer Bedeutung sind. Gleichzeitig wissen wir um die wissenschaftlichen Denkstile, die sich in bestimmten wissenschaftlichen Praktiken niederschlagen und damit auch eine kanonische Wirkung entfalten, u. a. auch auf die diagrammatischen Mittel, 
die in einer Disziplin zu einer bestimmten Zeit gängig sind (vgl. dazu Kapitel 3.2 und 3.3).

In der Diagrammatisch-Forschung spielen, so weit ich sehe, solche kulturellen Aspekte jedoch noch keine große Rolle. Zwar wird z. B. aus kunsthistorischer Sicht mit dem Stilbegriff operiert (z. B. Bredekamp et al. 2008): Tut man das, dann ist es wichtig, diagrammatische Ausdrucksformen mit kulturellen Praktiken in Verbindungen zu bringen, wie das beispielsweise Katja Mayer macht, indem sie Netzwerkforschung als „visual history“ betreibt (Mayer 2011). Auch ich versuchte in diesem Buch an verschiedenen Stellen den Zusammenhang von kulturellen Praktiken, technischen Möglichkeiten und dem Gebrauch von Diagrammen in der Linguistik zu untersuchen.

Es scheint mir jedoch noch einiges brach zu liegen, beispielsweise wenn ein Fokus auf Gender gelegt würde. Im Hinblick auf die Technikgeschichte ist beispielsweise inzwischen gut beschrieben, wie die weiblichen Programmiererinnen der 1950er-Jahre langsam aus dem Feld des Programmierens verschwunden sind (Thompson 2019). Inzwischen, vor dem Hintergrund neuer Coding Cultures, müssen damit verbundene Konstruktionen von Gender sicher neu untersucht werden - einerseits hat der Anteil von „women in tech“ wieder zugenommen, andererseits muss ein eigentlicher „Gender Bias“ festgestellt werden, der sich beispielsweise bei der Entwicklung von Systemen Künstlicher Intelligenz dramatisch auswirkt (Myers West et al. 2019). Code ist nie neutral. Wer also mit welchen Mitteln welche Technologien entwickelt, um - in unserem Fall - Daten zu transformieren und zu visualisieren, wirkt sich auf die Analyse der Daten aus. Bezieht man zusätzlich die historische Perspektive mit ein, wird die Komplexität der Kulturen der Diagrammatik und Visualisierung deutlich.

Die Geistes- und Sozialwissenschaften sind sicher ein besonders geeigneter Ort, um Visualisierungen nicht einfach als „Tool“ zu sehen, sondern die soziokulturellen Konstruktionen, die Ensembles von Praktiken und Techniken zu Tools machen, zu reflektieren.

\subsubsection{Hacking und Bricolage: Ausblicke einer transsemiotischen Linguistik}

An verschiedenen Stellen habe ich auf das Prinzip des Hacking-Paradigmas, auch in einem abstrakten Sinn, verwiesen, mit dem herrschende Denkstile hinterfragt werden können. Im Kontext der Software Studies spricht Goffey $(2014,35)$ von „Bricolage“, also von Tricks und Bastellösungen während des Programmierens, die aber oft hinter einer Oberfläche verschwinden (vgl. Kapitel 4.4.1). Das Gehäuse öffnen und das Innere verändern (hacken) und zu etwas Neuem rekombinieren (basteln) sind Praktiken, mit denen neue Perspektiven auf Gegenstände gewon- 
nen, manchmal aber auch neue Gegenstände überhaupt erzeugt werden. Das ist das Programm einer „Visuellen Linguistik“, wie in diesem Buch beschrieben ist.

Dieses Programm kann über das Visuelle hinaus gedacht werden: Die bisherigen Ausführungen haben bereits deutlich gemacht, dass ich selbst unter „Visueller Linguistik“ mehr verstehe, als grafische Darstellungen und dass Diagrammatik über die inskribierte Fläche hinaus geht. Auch mit Jägers Konzept der Transkriptivität, verstanden als semiotische Medientheorie, wird die abstraktere Perspektive deutlich (vgl. Kapitel 3.1). Insofern stellen die im Buch gemachten Überlegungen auch eine Grundlage für transsemiotische und multimodale Theoriebildung dar (Kress 2010; Seizov/Wildfeuer 2017). Dies wird hoffentlich auch an drei kleinen Beispielen unserer aktuellen Forschung deutlich:

\section{Digital Conversation Analysis and Feedback Table}

Im Anschluss an die Überlegungen zur Visualisierung von Gesprächen (Kapitel 8) entstand die Idee, räumliche Interaktion und Körperlichkeit noch stärker zu berücksichtigen. Ausgangspunkt ist ein runder Tisch, an dem mehrere Personen

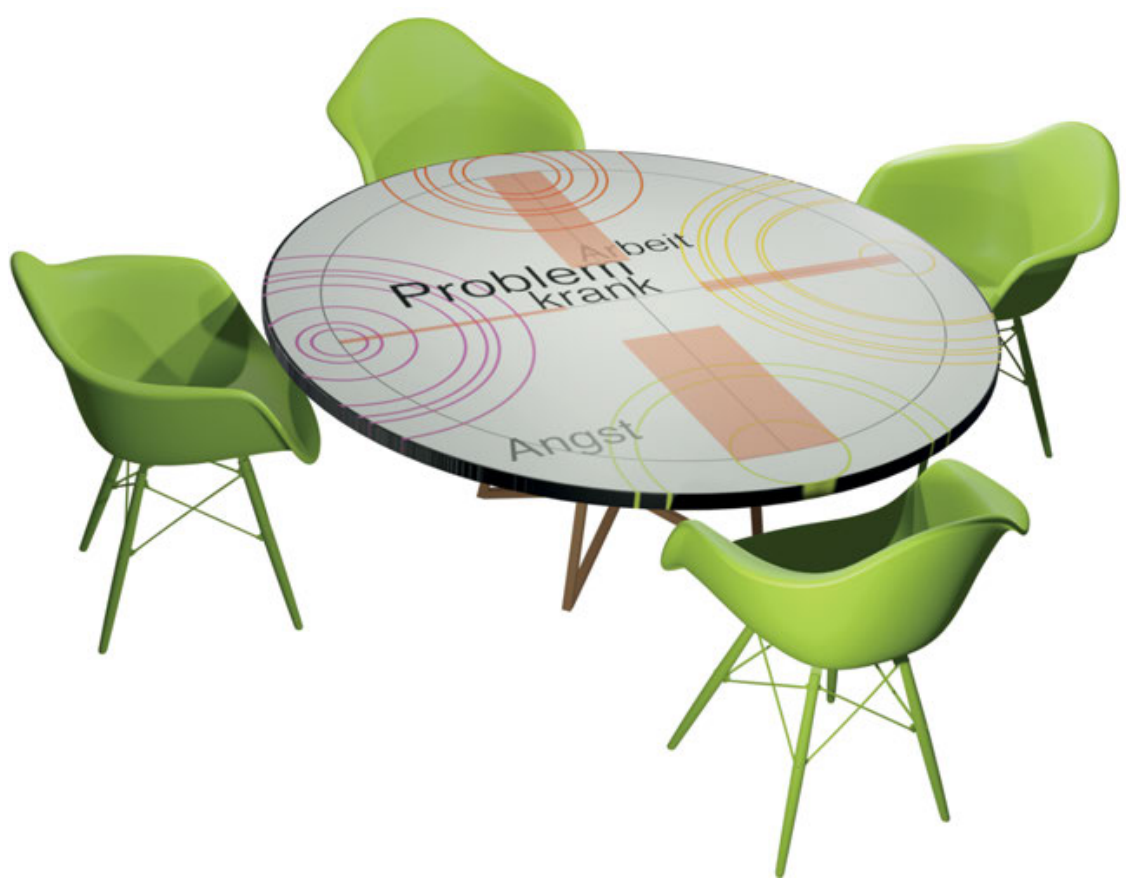

Abb. 111: Skizze des Digital Conversation Analysis and Feedback Table (Rendering: Maaike Kellenberger) 
sitzen und miteinander sprechen können. Über Mikrofone und Laryngographen (um zusätzlich ein besseres Signal direkt vom Kehlkopf zu haben) werden die Audiosignale erfasst und prozessiert. Dabei können automatisch mehrere Merkmale wie Stimmqualität, Intonation, Sprechgeschwindigkeit, Turnlänge etc. analysiert werden. Mittels Spracherkennung kann der Inhalt der Gespräche in Text konvertiert und computerlinguistisch ausgewertet werden. Der Tisch selber stellt gleichzeitig eine Projektionsfläche dar, so dass Gesprächsverhalten der Teilnehmenden live dargestellt werden kann (vgl. Abbildung 111). Wie bereits in Kapitel 8 dargestellt, kann durch die Visualisierung der Merkmale eine holistische Sicht auf die Gesprächsdynamik erzeugt werden. So kann sichtbar gemacht werden, wer gesprächsbeherrschend ist, welche Themen im Zentrum des Gesprächs stehen, was langsam an den Rand gedrängt oder gar ganz „vom Tisch fällt“.

Der Tisch ist weit mehr als ein Analyseinstrument für Gespräche, denn die visualisierten Metadaten zum aktuellen Gespräch werden wiederum eine Auswirkung auf die Gesprächsteilnehmenden haben - Metareflexion wird Teil der Gesprächsinteraktion. Gleichzeitig bietet der Tisch auch die Möglichkeit, im Sinne des Iconic Turns, die Visualisierung selber zu einem Erkenntnismedium werden zu lassen, indem der Visualisierungsprozess auch für die Teilnehmenden transparent gemacht wird (wenn man so will: sie können ihn hacken): Welche Metaphorik leitet uns, um Gesprächsdynamiken zu beschreiben und eben auch analytisch sichtbar zu machen? Ausdrücke wie „vom Tisch fallen“, „auf den Tisch hauen“ oder ein Gespräch „am runden Tisch“ können so reflektiert werden.

\section{Kommunikationsräume um 1900 als VR-Erfahrung}

Im Rahmen der Promotion von Larissa Schüller zu Telefonistinnen in den Telefonzentralen vor der Umstellung auf automatische Vermittlung, erproben wir mit einem erweiterten Team die Möglichkeit, Virtual Reality zu nutzen, um Telefonzentralen um 1900 als begehbare virtuelle Welt zu modellieren. ${ }^{102}$ Dabei geht es darum eine Art re-enactment, eine „spielerische Heuristik“ (Fickers 2015) zu ermöglichen, um die kommunikativen Bedingungen der Berufs der Telefonistin nachvollziehen zu können. Die Virtual-Reality-Anwendung ist als interaktive Erfahrung konzipiert: Es kommen Telefonanrufe herein, die unter Beachtung des verlangten Protokolls abgearbeitet werden müssen, links und rechts sitzen weitere Telefonistinnen, die Geräuschkulisse und Atmosphäre der Zentrale kann damit simuliert werden. Gezielt können verschiedene Parameter, die die Arbeit

102 Larissa Schüller leitet das Projekt, in dem Maaike Kellenberger, Christoph Hottiger, Patrick Jost und der Autor arbeiten. 
wahrscheinlich beeinflussen, geändert werden: Ausgestaltung des Arbeitsplatzes, Geräte, Typ der Telefonzentrale, Anzahl der Anrufe etc. Damit wird es möglich, die Arbeitssituation der Telefonistinnen auch körperlich nachvollziehen zu können und damit analysierbar zu machen.

Einerseits ist auch dieses Projekt ein Experiment, um den Kommunikationsberuf der Telefonistinnen linguistisch zu analysieren. Andererseits liegt das Interesse jedoch genau so stark am Instrument der Virtual Reality selber: Es ist kein Konsuminstrument, kein Mittel zum Zweck, sondern Teil des Experiments, um herauszufinden, welche diagrammatischen Eigenschaften es mitbringt, welchen Denkstilen es folgt (eine VR-Brille wird primär als Konsum- und vor allem Gaming-Device vermarktet) und wie sich der Untersuchungsgegenstand mit den Möglichkeiten von VR neu konstituiert.

\section{Von der Visualisierung zum Interface: Goettherina}

Sehr zufällig als Spielerei ist der Gedichtautomat „Goettherina“ entstanden, ein Objekt aus Holz, Arduino-Bord (Einplatinen-Rechner), Servo, LEDs, Liquid Cristal Display, Schaltern, Thermoprinter, Lautsprecher, Tastatur, Python-Programm

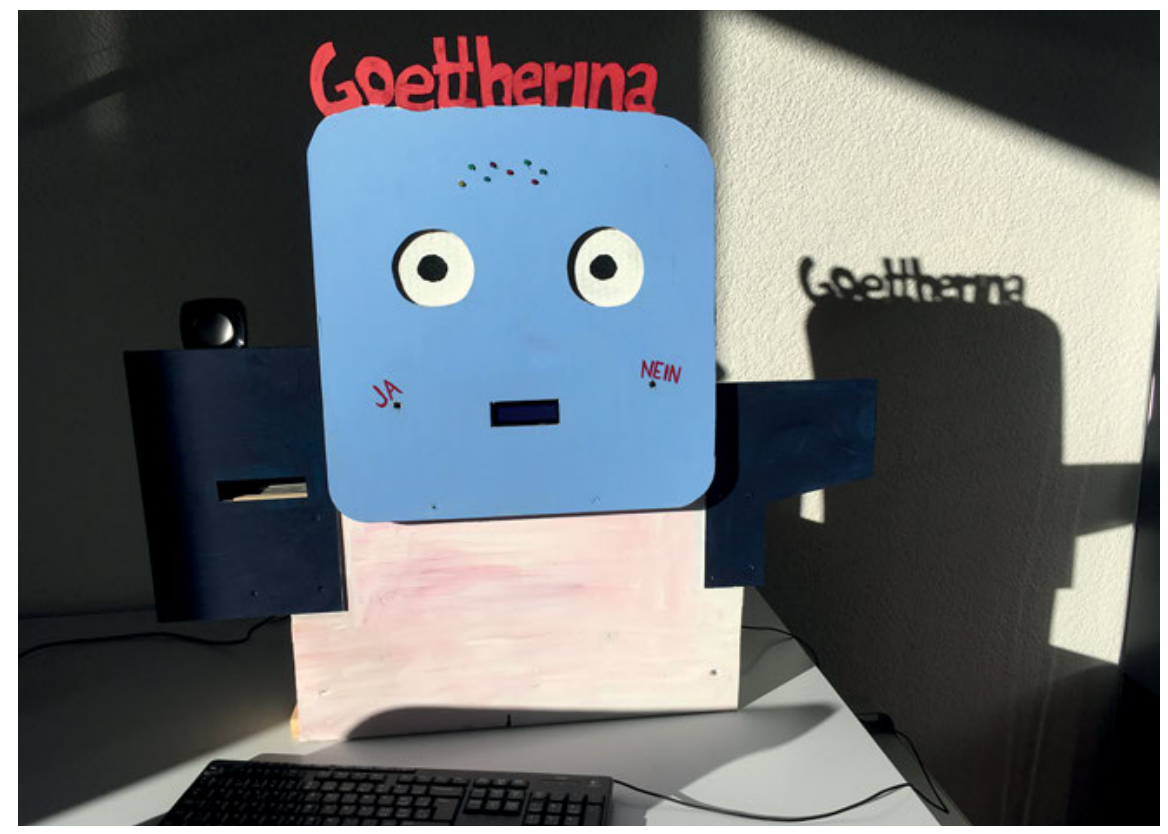

Abb. 112: Gedichtautomat „Goettherina“ (vgl. www.bubenhofer.com/visuallinguistics/ für eine Demo) 
und Korpusdaten. ${ }^{103}$ Der Automat erstellt nach Eingabe eines Wortes automatisch ein Gedicht, wobei als Datengrundlage ein Textkorpus aus deutschsprachigen Gedichten der letzten Jahrhunderte und Zeitungstexten (darunter Sportartikelüberschriften) dient. Das Python-Programm versucht nach einem bestimmten Reimschema sich reimende Zeilen zu finden. Der Automat mutet Roboter-ähnlich an, da er (bzw. sie) spricht, die Augen bewegt, blinkt und Textausgaben auf dem Minidisplay erzeugen kann. Das Gedicht kann zudem auf dem Thermodrucker als „Kassenbeleg“ ausgegeben werden.

Was ist nun das wissenschaftliche Interesse an Goettherina? Zunächst ist es sowohl linguistisch als auch literaturwissenschaftlich interessant, einen Algorithmus zu entwickeln, mit dem zufällige Gedichte erstellt werden können. Das automatische Erstellen von Gedichten hat dabei eine lange Tradition (Reither 2015) und wird z. B. auch gezielt didaktisch im Gymnasialunterricht eingesetzt (Wampfler 2017, 141).

Aus der Sicht einer „Visuellen Linguistik“ jedoch kommen diagrammatische und transsemiotische Aspekte hinzu: Das „Dichten“ ist ein Prozess der Transkription und dabei auch diagrammatischen Umordnung von Textdaten, allerdings in Kombination mit der Interaktion einer Person, die den Automaten in Gang setzt und beeinflusst. Die Visualisierung des Gedichtes selber, aber eben auch der Prozesse, die zum Gedicht führen, ist eine Mischung aus sinnlich wahrnehmbaren Elementen: Blinken, Augenbewegung, Drucken, Stimme. Der Automat operiert nicht nur digital, sondern auch physisch, indem ein Servo in Bewegung gesetzt wird, der die Pappe für die Augen bewegt, gedruckt und geblinkt wird, er funktioniert also multimedial und wirkt transsemiotisch, indem verschiedene Zeichensysteme miteinander interagieren.

Mit der Programmierung, dem Basteln und der Nutzung des Automaten geht eine Reflexion über die verwendeten Techniken einher: Der Arduino-Computer bringt eine umfassende Technokultur des Hackens und Bastelns nach bestimmten Prinzipien (Open Source) mit, die synthetische, weibliche Stimme aktualisiert die Frage nach dem Zusammenhang von Gender, Computer und Funktion neu und grundsätzlich wird die Idee des Interfaces zwischen Mensch und Maschine problematisiert.

Damit möchte ich die lange Reise vom „Kreis als Diagramm oder Bild“(Abbildung 1) beschließen. Mein Ziel lag darin, grundsätzlich über die Genese, Funktion und Kategorisierung von Diagrammen in der Linguistik nachzudenken. Dabei eröffnete sich mir eine komplexe Welt von Visualisierung, Medialität, Digitalität und

103 Vgl. www.bubenhofer.com/visuallinguistics/. 
Wissensgeschichte, die nun hoffentlich nicht nur einen genaueren Blick auf Diagramme in der Linguistik ermöglicht, sondern auch zu neuen Ideen und Experimenten anstiftet. 
